\title{
UTILIDAD DEL LÁSER DE HOLMIUM:YAG EN LA SECCIÓN ENDOSCÓPICA DE LA ESTENOSIS URETERAL
}

Pablo Garrido Abad, Inmaculada Fernández González, Almudena Coloma Del Peso, Manuel Fernández Arjonal, Gloria Bocardo Fajardo, Milagros Jiménez Glaves', Luis Miguel Herranz Fernández', Álvaro Serrano Pascual2, Lorenzo Herrero Torres e Ignacio Pereira Sanz.

Servicio de Urología del Hospital de La Princesa. Madrid. España.

'Servicio de Urología del Hospital del Henares. Coslada. Madrid. España.

2 Servicio de Urología del Hospital de Guadalajara. Guadalajara. España.

Resumen.- La incidencia de la estenosis ureteral es frecuente en nuestro medio. En los últimos tiempos, debido al masivo empleo de técnicas endourológicas, ha aumentado su incidencia.

Su etiología representa un factor decisivo para el resultado final del tratamiento, pero existen además características comunes a todas las estenosis que influyen de forma muy importante en el éxito final: tiempo de evolución, longitud de la estenosis, localización de la misma y función de la unidad renal afecta.

En los últimos años se ha incrementado la utilización de diferentes técnicas endourológicas para el tratamiento de las estenosis del aparato urinario superior, que vienen a sustituir a la técnica abierta tradicional. La endoureterotomía con láser de Holmium:YAG presenta ventajas frente a otras técnicas endourológicas empleadas, ya que permite una incisión precisa con visión directa sobre la estenosis ureteral. Además, con las fibras de láser se cosigue una flexibilidad/deflexión del ureteroscopio, que permite alcanzar, en la inmensa mayoría de los casos, la zona estenótica.

A la hora de realizar la incisión sobre la pared ureteral, ésta debe ser completa, actuándose sobre todas las capas del uréter hasta visualizar la grasa periureteral, teniendo siempre en cuenta las relaciones del uréter con las estructuras vecinas, especialmente las vasculares, para evitar lesionarlas.

Su efectividad y fácil manejo, permiten obtener una elevada tasa de éxitos, con resolución de la patología estenótica, y una muy baja tasa de complicaciones. Por todo ello, debe incluirse a la endoureterotomía retrógrada con láser de Holmium:YAG en la primera línea del tratamiento de las estenosis ureterales de tipo benigno.

Palabras clave: Estenosis ureteral. Láser.

Summary.- OBJECTIVES: The incidence of ureteral stenosis is frequent in our environment. Lately, due to the massive use of endourological techniques its incidence has increased.

Etiology represents a decisive factor for the final result of treatment, but there are also common characteristics to all stenosis that influence very importantly the final success: time of evolution, length of the stenosis, side and function of the affected renal unit. 
Over the last years, the use of endourological techniques for the treatment of upper urinary tract stenosis, that substitute the traditional open technique, have increased. Holmium:YAG laser endoureterotomy presents advantages in comparison with other endourological techniques, because it enables a precise incision with direct vision of the ureteral stenosis. Moreover, with laser fibers ureteroscopes achieve a degree of flexibility/ deflection that enables us to reach in most cases the stenotic area.

At the time of incision of the ureteral wall, it should be complete, acting on all ureteral layers down to the periureteral fat, always having in mind the anatomic relationships of the ureter with neighbour structures, mainly vascular, to avoid injuries.

Its effectiveness and easy management permits a high success rate, with resolution of the stenosis and a very low complication rate. After all the anterior, holmium laser retrograde endoureterotomy should be included as a first line treatment for benign ureteral stenosis.

Keywords: Ureteral stenosis. Laser.

\section{INTRODUCCIÓN}

Una estenosis ureteral se define como un estrechamiento del uréter que causa una obstrucción funcional. (1) La incidencia de la estenosis ureteral es frecuente en nuestro medio. Debido al masivo empleo de técnicas endourológicas ha aumentado su incidencia. Se estima que tras la realización de una ureteroscopia aparece la estenosis entre un 7-15\%, en función del calibre y de su condición de flexibilidad (2). Además, son muy prevalentes también las estenosis de la unión pieloureteral y las producidas en la anastomosis ureterointestinal de las derivaciones urinarias $(4-22 \%)$, variando según la técnica quirúrgica $(3,4)$.

\section{ETIOLOGÍA}

Las estenosis del aparato urinario superior pueden ser congénitas 0 adquiridas. Las de tipo congénito están comúnmente localizadas en la unión pieloureteral. Con la excepción de esta obstrucción primaria de la unión pieloureteral, la mayoría de las estenosis ureterales son adquiridas y usualmente son iatrogénicas. La causa más común de iatrogenia es la lesión durante maniobras quirúrgicas abiertas, endoscópicas o laparoscópicas. Las estenosis ureterales adquiridas y no iatrogénicas incluyen las que se producen tras la expulsión de un cálculo ureteral y debidas a la afectación inflamatoria crónica, como en el caso de la tuberculosis. (5) Se pueden dividir también en estenosis de tipo benigno y maligno. Las de tipo benigno se pueden subdividir a su vez en isquémicas (ocurren tras cirugía abierta y radioterapia) y no isquémicas (ocurren tras el paso del cálculo o debida a una anormalidad congénita). Las estenosis provocadas por maniobras endoscópicas pueden ser tanto de tipo isquémico como no isquémico según el mecanismo de la lesión. Las de tipo maligno son las causadas por la recidiva de un tumor primario o por compresión extrínseca de ese tumor (5).

Su etiología representa un factor decisivo para el resultado final del tratamiento, pero existen además características comunes a todas las estenosis que influyen de forma muy importante en el éxito final: tiempo de evolución, longitud de la estenosis, localización de la misma y función de la unidad renal afecta. (1)

\section{DIAGNÓSTICO}

Los síntomas en presencia de una estenosis ureteral pueden incluir dolor lumbar, sordo o agudo, pielonefritis, urosepsis, litiasis renal, hematuria o uremia. En otros casos, el paciente puede presentar hiperazoemia asintomática (6).

El diagnóstico de estenosis ureteral se realiza mediante estudios de imágenes, como la urografía intravenosa (Figuras 1A, 1B y 1C), la ureteropielografía retrógrada, la pielografía o la gammagrafía renal. La ecografía renal tambíen puede ser útil. (1) Con estos estudios determinamos la localización de la estenosis y su longitud asi como la función renal ipsilateral.

En caso de decidir la realización de una endoureterotomía se realiza una Angio Tomografía Axial Computerizada (Angio-TAC) con reconstrucción para ver la relación de la estenosis con estructuras vasculares vecinas (Figuras $2 \mathrm{~A}, 2 \mathrm{~B}$ y $2 \mathrm{C}$ ) y determinar así el lugar de la sección para evitar el daño vascular.

\section{TRATAMIENTO}

En la década de los años 40, Davis se centró en la fisiopatología de la endoureterotomía intubada basándose en experimentos animales. Esta investigación se ha convertido en la base del tratamiento de las estenosis ureterales $(7,8)$.

En los últimos años se ha incrementado la utilización de diferentes técnicas endourológicas para el tratamiento de las estenosis del aparato urinario superior (balón de dilatación, endoincisión con electrocauterio, Acucise ${ }^{\mathrm{TM}}$, y cuchillete frío). Aunque los resultados a largo plazo pueden no ser tan satisfactorios o duraderos como los que ofrece la cirugía tradicional abierta, estas técnicas pueden ser realizadas con menor morbilidad.

Se han utilizado también varios tipos de láser para tratar esta patología, como el Nd:YAG, KTP y el Homium:YAG. Este último parece que consigue mejores resultados, ya que posee tanto propiedades de corte como de coagulación. El Holmium:YAG, es un láser de infrarrojos medio, de estado sólido, con energía pulsada y una longitud de onda de $2100 \mathrm{~nm}$. Se puede insertar a través de un endoscopio rígido o flexible, existiendo además varios calibres de fibras láser (200, 365, 500 y 1000 pm). 


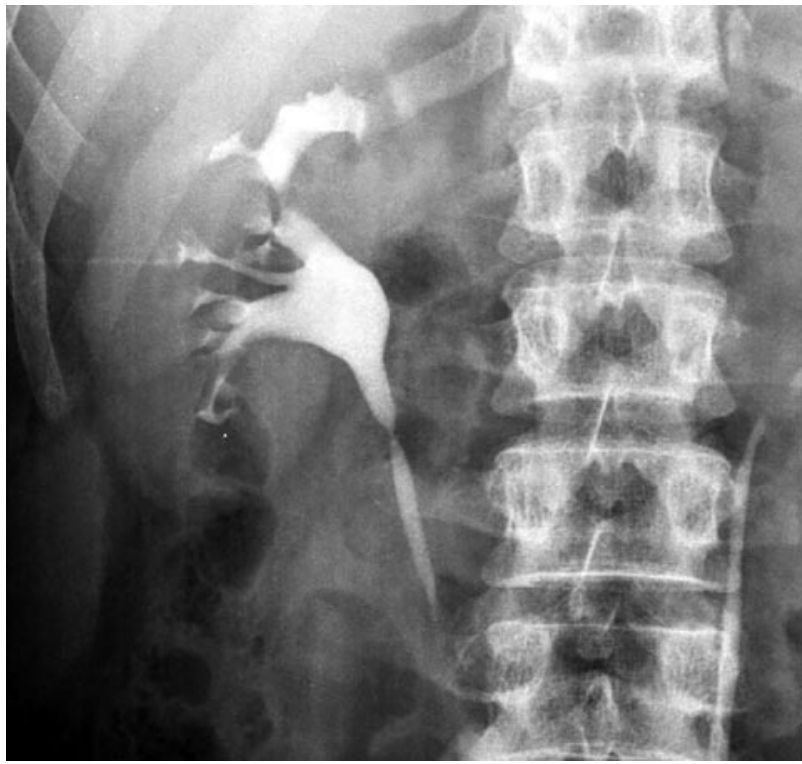

FIGURA IA. UIV. Estenosis ureteral lumbar derecha.

Técnica de realización de la endoureterotomía con láser Holmium:YAG

La endoureterotomía con láser de Holmium:YAG presenta ventajas frente a otras técnicas endourológicas empleadas, ya que permite una incisión precisa (profundidad de corte de $0.4 \mathrm{~mm}$.), con visión directa sobre la estenosis ureteral. Además de la sección, origina una vaporización del tejido cicatricial con un daño periférico termal escaso. Con las fibras de láser se consigue una flexibili$\mathrm{dad} /$ deflexión del ureteroscopio, que permite alcanzar en la inmensa mayoría de los casos la zona estenótica (9).

Con el paciente en posición de litotomía modificada y con anestesia raquídea, se procede en primer lugar a la realización de una ureteropielografía retrógrada (Figuras $3 \mathrm{~A}$ y $3 \mathrm{~B}$ ). Es fundamental tutorizar el segmento estenótico con una guía (Figuras $4 \mathrm{~A}$ y $4 \mathrm{~B}$ ). De forma habitual se procede a dilatación del trayecto intramural del uréter con balón de alta presión. Se accede a la zona de estenosis mediante el ureteroscopio semirrígido, utilizando el ureteroscopio flexible en los casos en los que esto no sea posible (Figura 5). Se procede a la sección endoscópica teniendo en cuenta la localización de la estenosis. En el uréter lumbar la sección se realiza en la zona ínfero-externa para evitar dañar los vasos gonadales; en uréter sacro a las 12 horas del horario cistoscópico para no lesionar los vasos ilíacos; en uréter pelviano alto en la zona súperointerna por los vasos ilíacos internos; en uréter pelviano en la mujer en la zona interna y a nivel del uréter yuxtavesical a las 12 horas (Figuras 6 y 7). La sección debe afectar a toda la pared ureteral hasta llegar a grasa. Este aspecto se comprueba con el extravasado que se visualiza con la ureteropielografía de control al finalizar el procedimiento (Figuras $8 a$ y $8 b$ ). Posteriormente se deja un catéter doble J calibre $7 \mathrm{~F}$ intubando la zona seccionada durante 6 semanas. A los tres meses se realiza UIV de control (Figura 9).

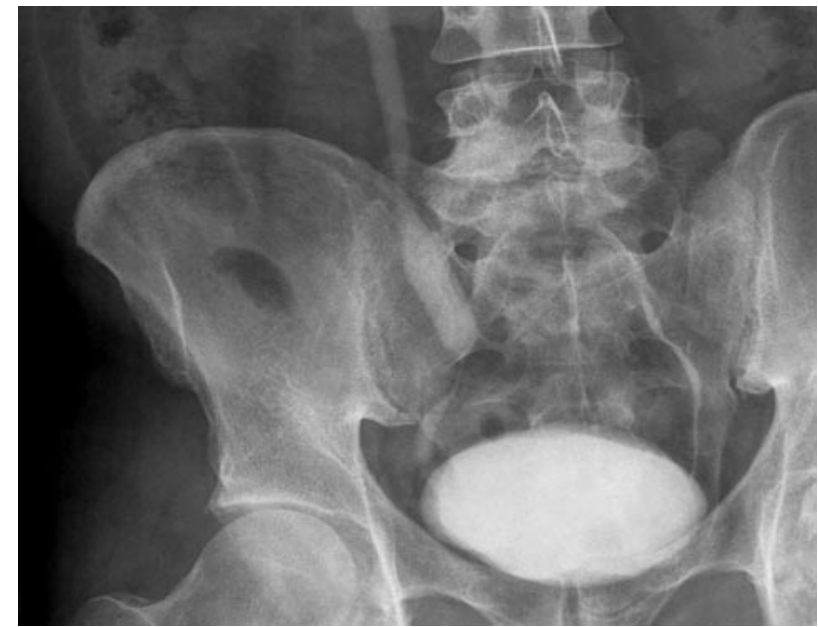

FIGURA 1B. UIV. Estenosis de uréter sacro derecho.

Nuestro grupo tiene experiencia en la ureteroscopia para el tratamiento de las estenosis ureteral de causa benigna con 5 casos de estenosis del tracto urinario superior. Se trata de 4 varones y 1 mujer, con una edad media de 48.3 años. Las estenosis se presentaron en diferentes localizaciones: uréter lumbar derecho (2), uréter lumbar izquierdo (1), uréter sacro derecho (1) y uréter pelviano izquierdo (1). La etiología de la estenosis era variable: congénita (1), secundaria a litiasis (3) y también secundaria a tuberculosis genitourinaria (1). En todos los pacientes la longitud de la estenosis fue menor de $2 \mathrm{~cm}$. En la totalidad de los casos se utilizó el láser VersaPulse ${ }^{\circledR}$ PowerSuite ${ }^{T M}$ (Lumenis, Inc.) de 100 vatios y un ureteroscopio semirrigido y fibra de láser Holmium:YAG de 500 micras con una

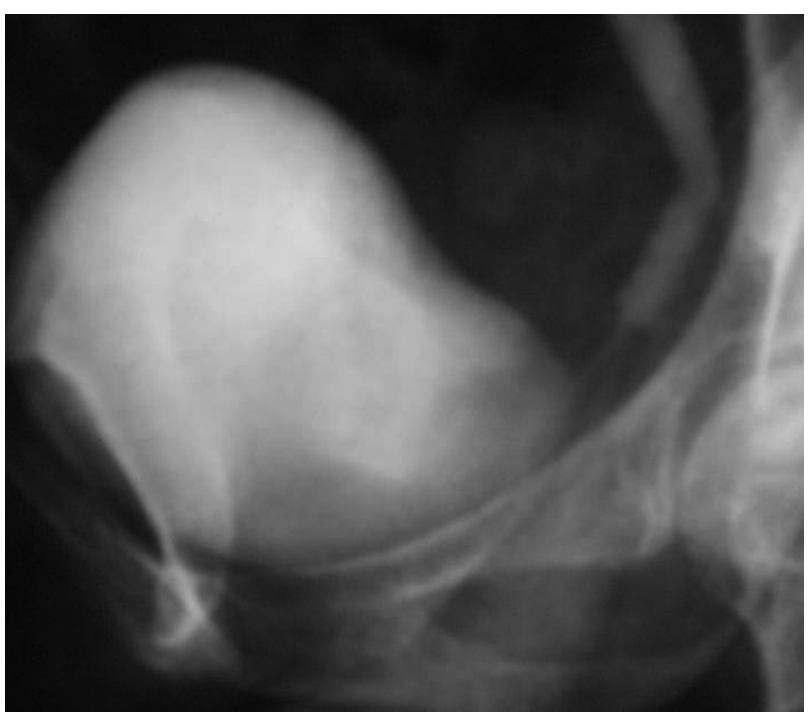

FIGURA 1C. UIV. Estenosis de uréter distal izquierdo. 


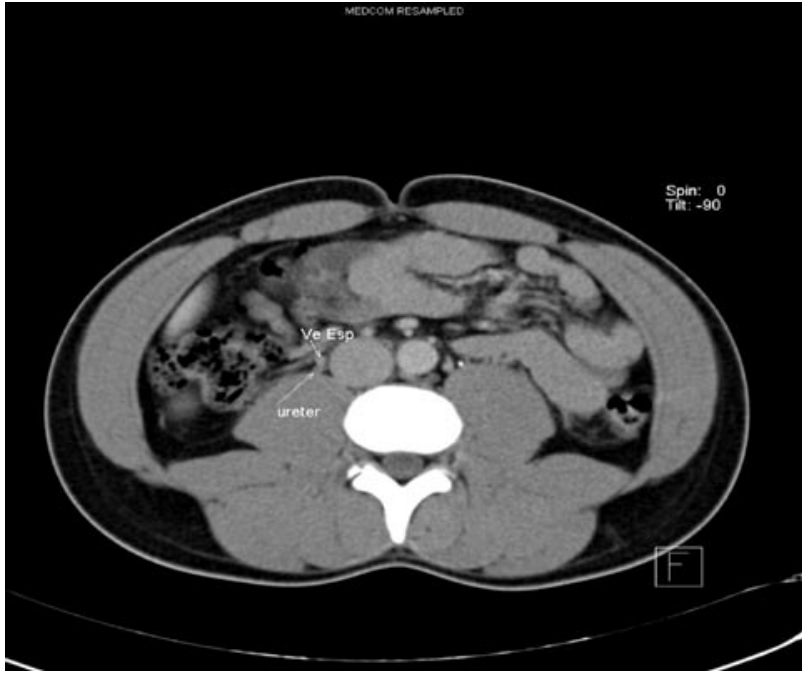

FIGURA 2A. TAC (corte transversal). Relación entre uréter y vena espermática en lado derecho.

potencia de 1,2 julios y una frecuencia de 10 Hertzios. La tasa de éxito a largo plazo (> 1 año) ha sido del $80 \%$, fracasando la técnica en un paciente con una función renal ipsilateral menor del $20 \%$, pero que permanece asintomático en la actualidad.

\section{DISCUSIÓN}

En lo referente a la estenosis ureteral, se han publicado tasas de éxito similares o superiores con la endoureterotomía con láser de Holmium:YAG, que con otras técnicas mínimamente invasivas. En una revisión multicéntrica realizada por Lane y cols. (10) se informa de una tasa de éxito global ligeramente superior al $75 \%$ de los casos, con un seguimiento medio de 36 meses. Otras series, más recientes, reportan incluso resultados superiores, con hasta un $88 \%$ de éxito a los 60 meses $(11,12,13)$. Se ha empleado con muy buenos resultados y excelente tolerancia también en la edad pediátrica, no siendo necesario, en la mayoría de estos casos, la colocación de stent preoperatorio, ni la dilatación intraoperatoria $(14,15)$. La necesidad de dilatación con balón neumático del orificio ureteral previa a la introducción del ureteroscopio es controvertida. Algunos autores incluso lo asocian con una mayor tasa de complicaciones. En caso de emplear un ureteroscopio semirígido, la dilatación previa, puede facilitarnos el acceso y la movilidad del mismo durante la intervención.

Durante la fase inicial del postoperatorio, se deben realizar técnicas de diagnóstico por imagen para determinar si el procedimiento ha tenido éxito. Si el paciente es portador de una sonda de nefrostomía, 2 a 3 semanas después de la retirada del catéter ureteral, se debe realizar un nefrostograma anterógrado y un renograma (DTPA). Si persiste la obstrucción, se mantiene la nefrostomía para drenaje por gravedad y se repiten las gammagrafías a las 3 ó 4 semanas, ya que el edema puede causar una obstrucción inicial que posteriormente se resuelve. Si el paciente no es portador de sonda de nefrostomía, se realiza un re-

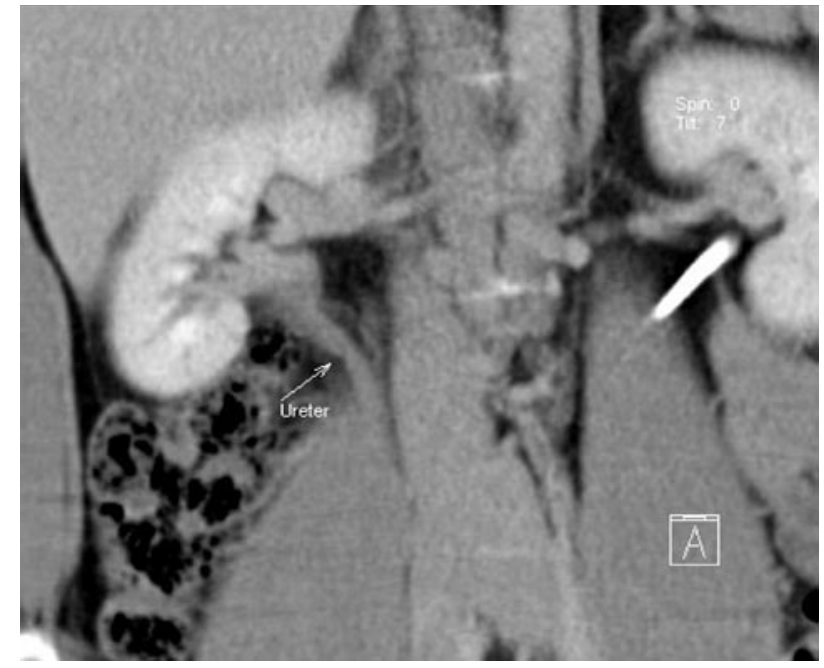

FIGURA 2B. TAC (corte coronal). Uréter derecho.

nograma DTPA o una urografía intravenosa 2 a 3 semanas después de la retirada de la endoprótesis. Si existe obstrucción se debe colocar sonda de nefrostomía y repetir el renograma DTPA o un nefrostograma anterógrado (1).

Para el seguimiento tardío se realiza urografía y/ o ecografía abdominal en 3-6 meses (1).

Observamos que con frecuencia, los casos fallidos de la técnica, aparecen de forma precoz lantes de los 3 meses) (10).

Su etiología representa un factor decisivo para el resultado final del tratamiento, pero existen además características comunes a todas las estenosis que influyen de for-

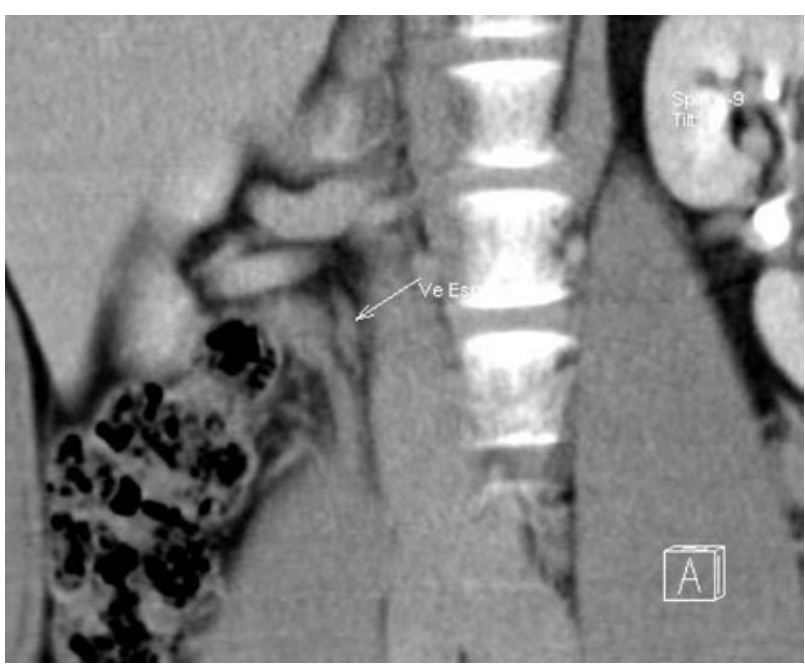

FIGURA 2C. TAC (corte coronal). Uréter derecho y su relación con la vena espermática. 


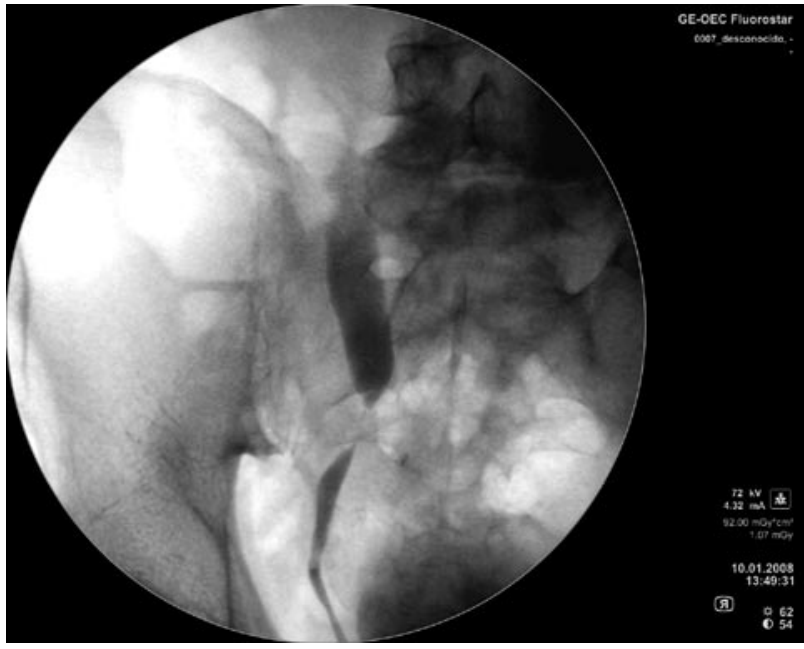

FIGURA 3A. Ureteropielografía retrógrada. Estenosis uréter sacro derecho.

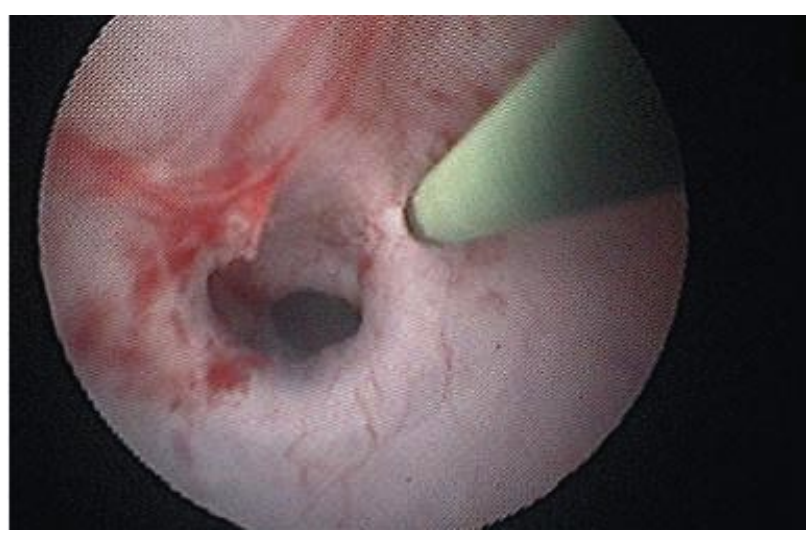

FIGURA 4A. Imagen endoscópica de la estenosis ureteral previa al paso de la guía de seguridad.

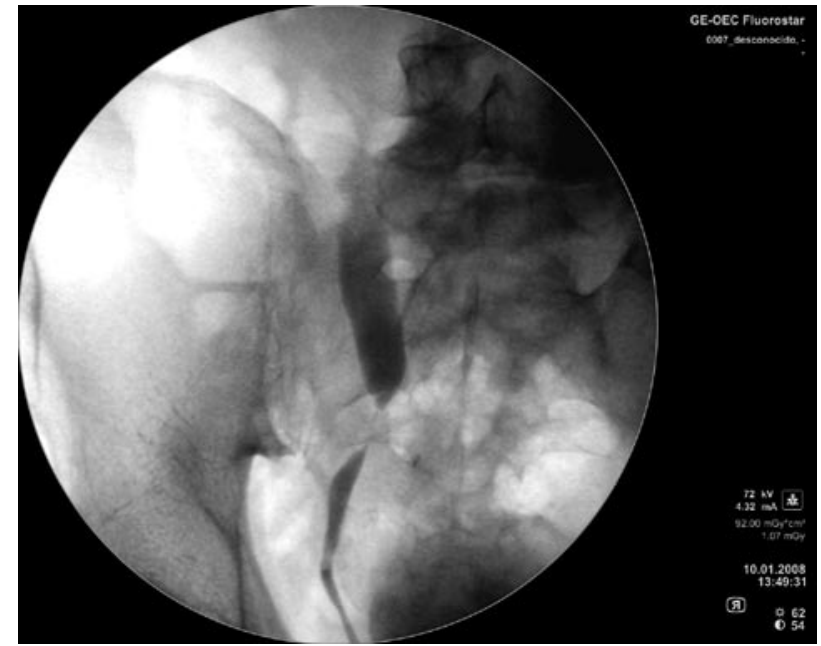

FIGURA 3B. Ureteropielografía retrógrada. Estenosis uréter lumbar derecho.

ma muy importante en el éxito final: tiempo de evolución, longitud de la estenosis, localización de la misma y función de la unidad renal afecta (1).

Las estenosis de causa no isquémica responden mejor al tratamiento, obteniéndose una tasa de éxito del $90 \%$ a los 3 años con endoureterotomía, en comparación con la tasa del $69 \%$ para estenosis isquémicas. Las estenosis causadas por carcinomas recurrentes o por compresión extrínseca (fibrosis retroperitoneal, linfoma) no responden bien al tratamiento endoscópico y con frecuencia se tratan mejor con un procedimiento quirúrgico abierto o laparoscópico (1). Suelen ser lesiones: de mayor longitud y tiempo de evolución, de localización proximal, sobre los que se
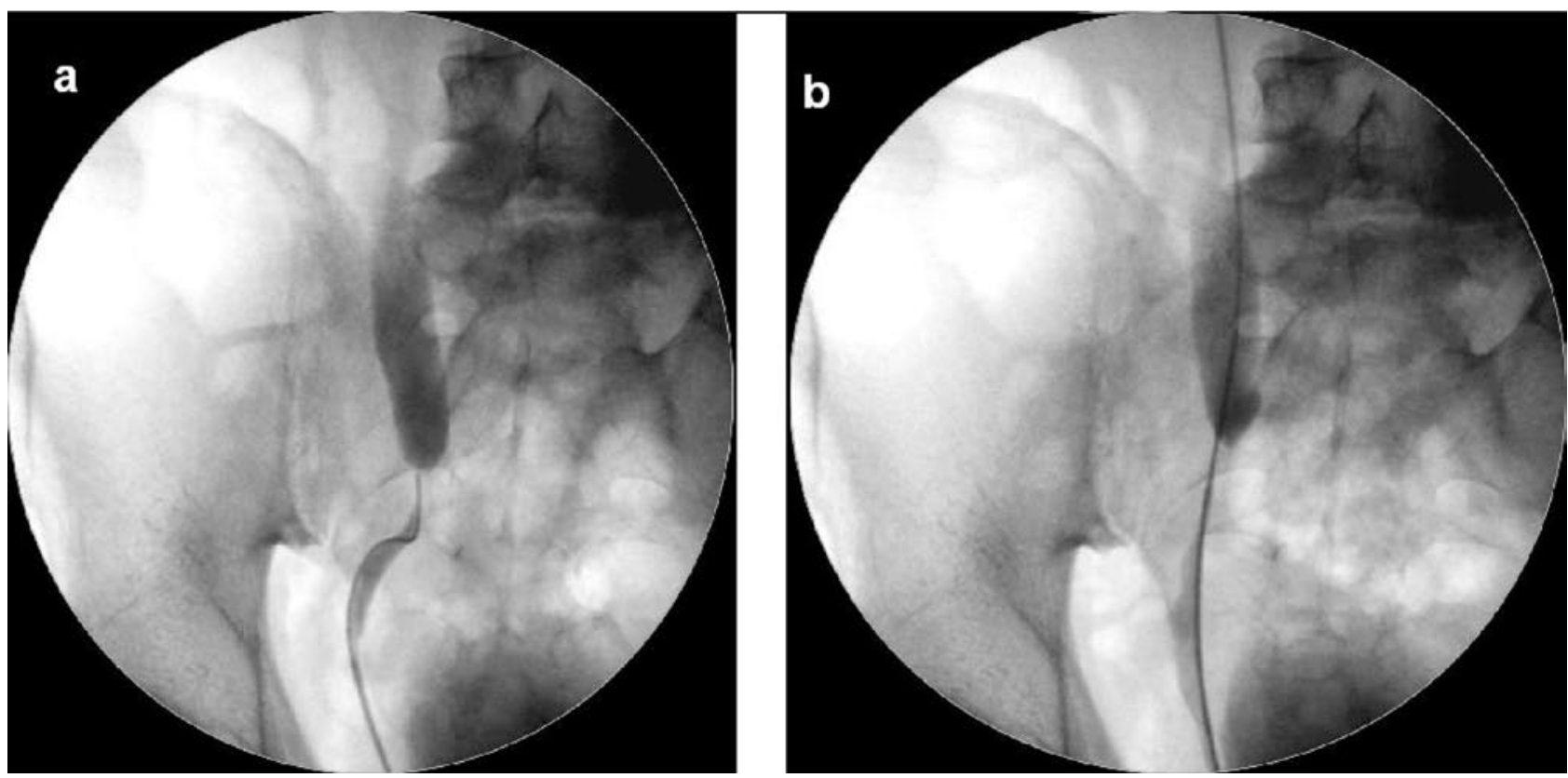

4B. Imagen en el amplificador de imágenes de la tutorización con guía del segmento estenótico ureteral derecho. 


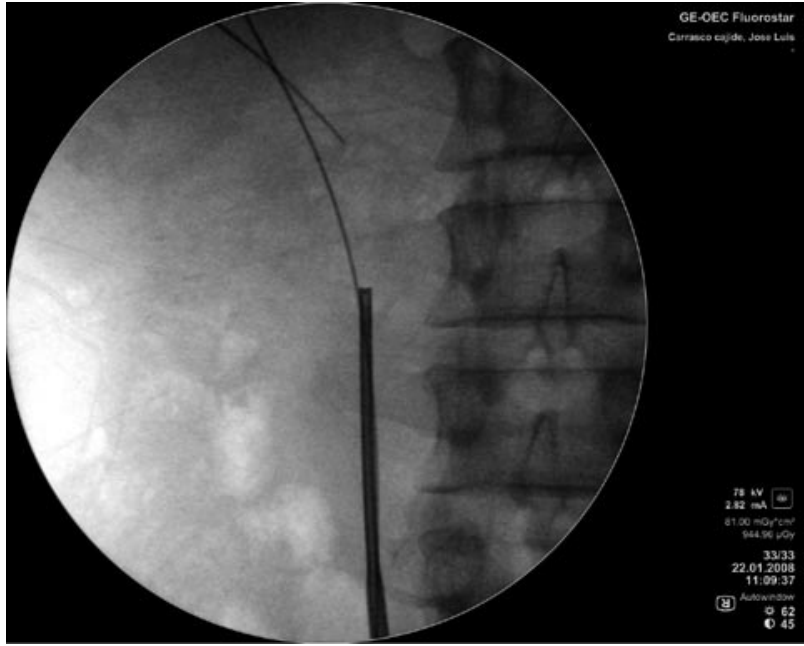

FIGURA 5. Imagen del control del amplificador de imágenes donde se observa el ureteroscopio semirrígido a nivel de la estenosis del uréter lumbar derecho.

han realizado intervenciones previas sin éxito, o con una mala funcionalidad asociada de la unidad renal afecta.

El tiempo de evolución de la estenosis ha demostrado ser inversamente proporcional a la tasa de éxito. Existen menos posibilidades de tener éxito mediante un tratamiento endoscópico cuando se conoce que la estenosis existe desde hace 6 a 24 meses, en comparación con estenosis de menor duración $(3,5)$.

Las estenosis cortas, especialmente las que miden menos de $1 \mathrm{~cm}$, responden mejor que las más largas. Las

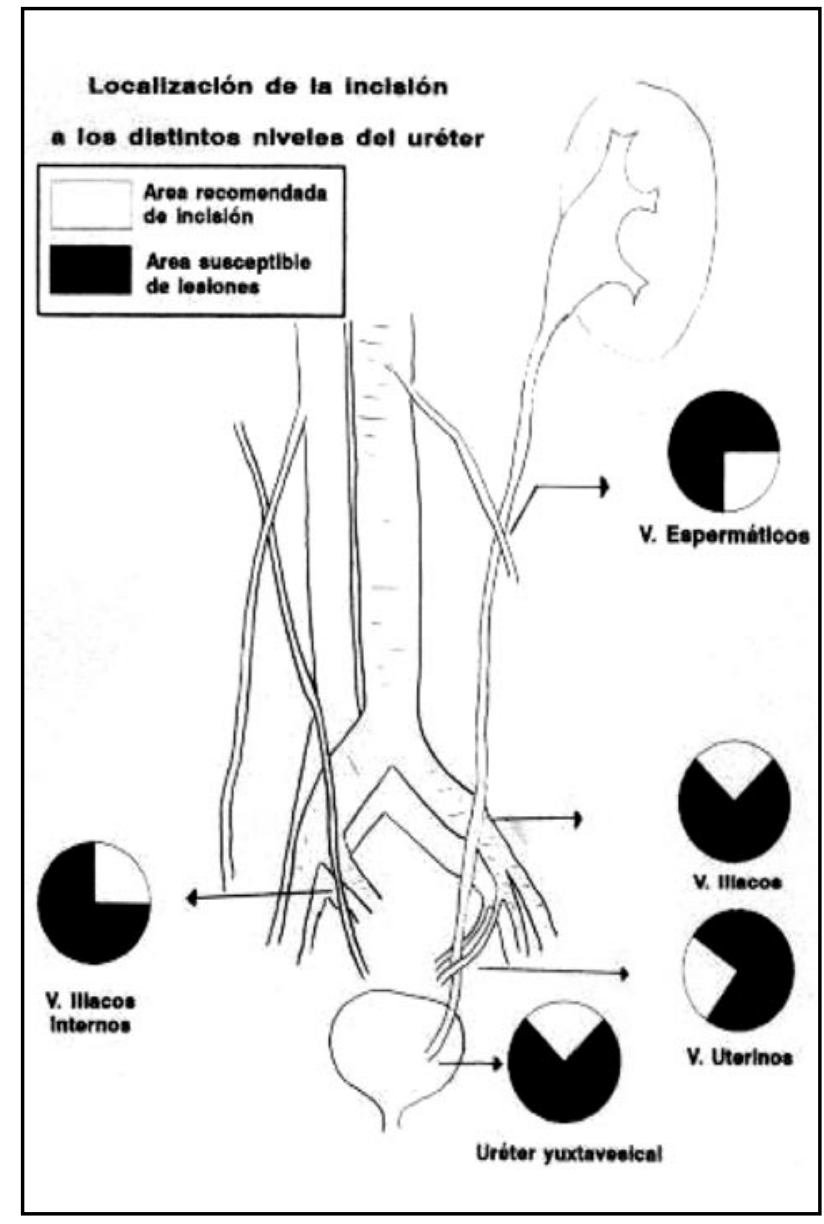

FIGURA 6. Lugar de la endoincisión ureteral en función de la localización de la estenosis.
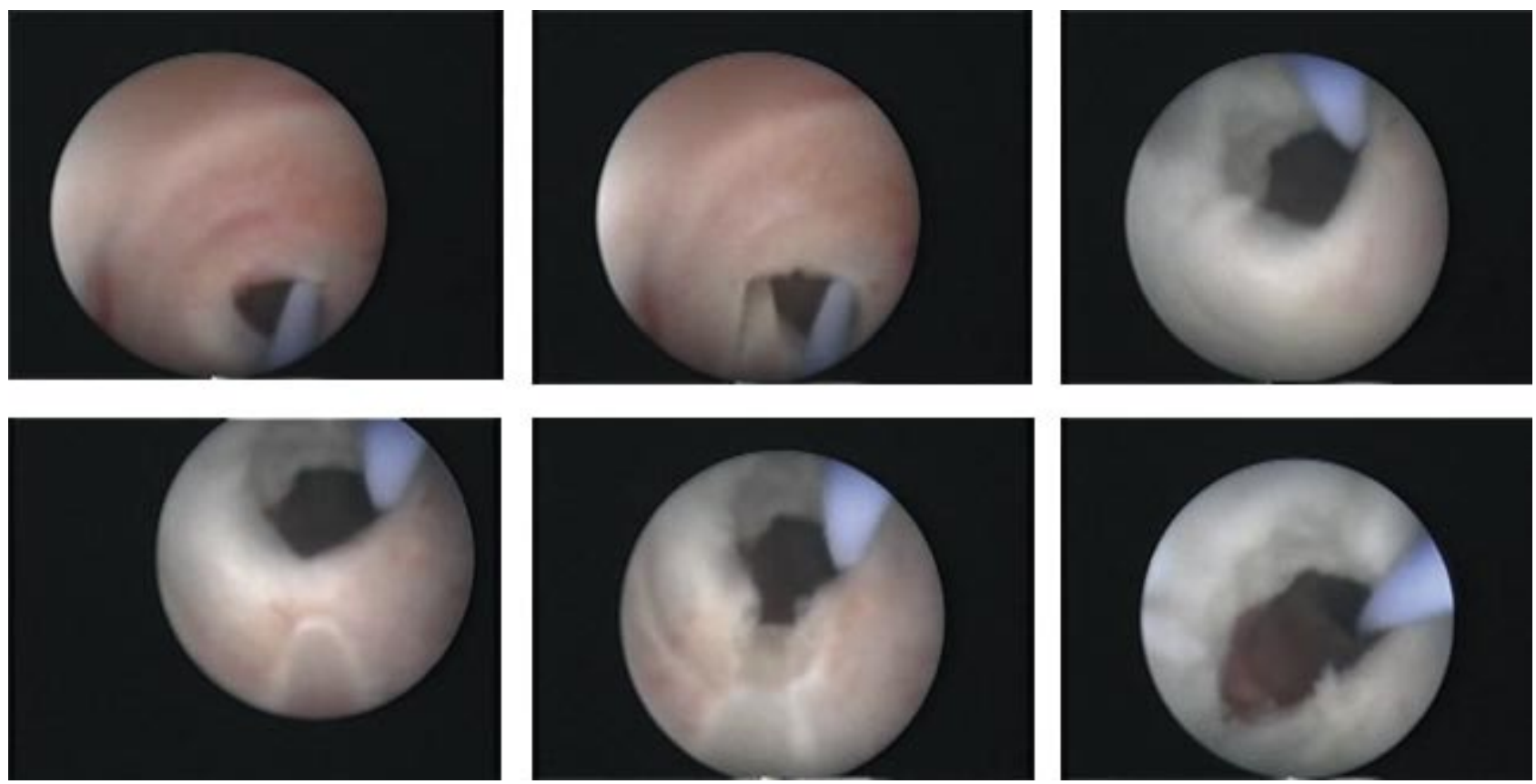

FIGURA 7. Imagenes endoscópicas de una endoincisión del uréter lumbar derecho a nivel infero-externo. 

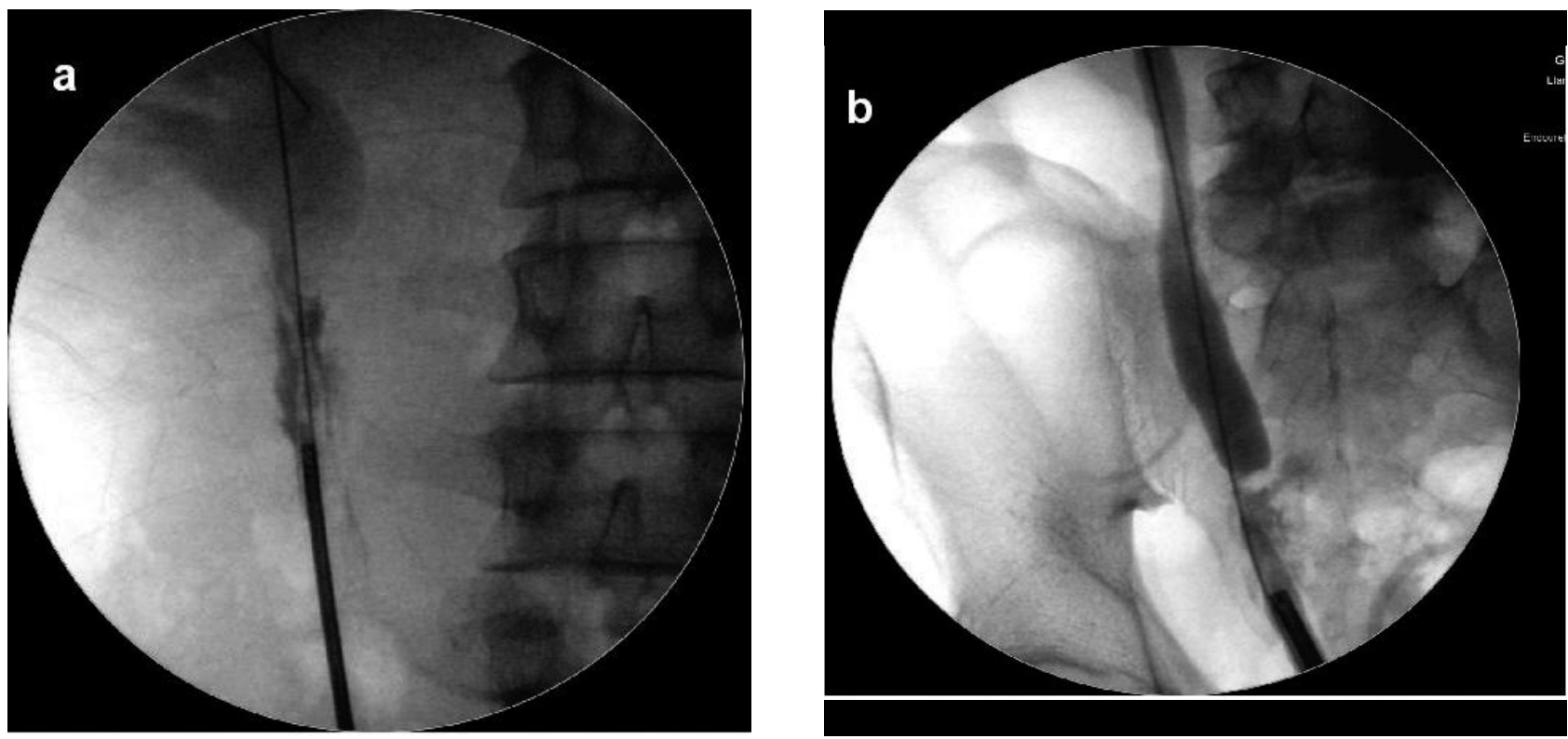

FIGURA 8. En el control del amplificador de imágenes se visualiza la extravasación del material de contraste al finalizar la sección de estenosis ureteral derecha.

estenosis más largas y completamente obstruidas tienen menos probabilidad de éxito con una endoureterotomía (3).

En cuanto a su localización, para las estenosis ureterales benignas, las localizaciones distales y proximales tienen mejor tasa de cicatrización en comparación con el tercio medial del uréter (5).

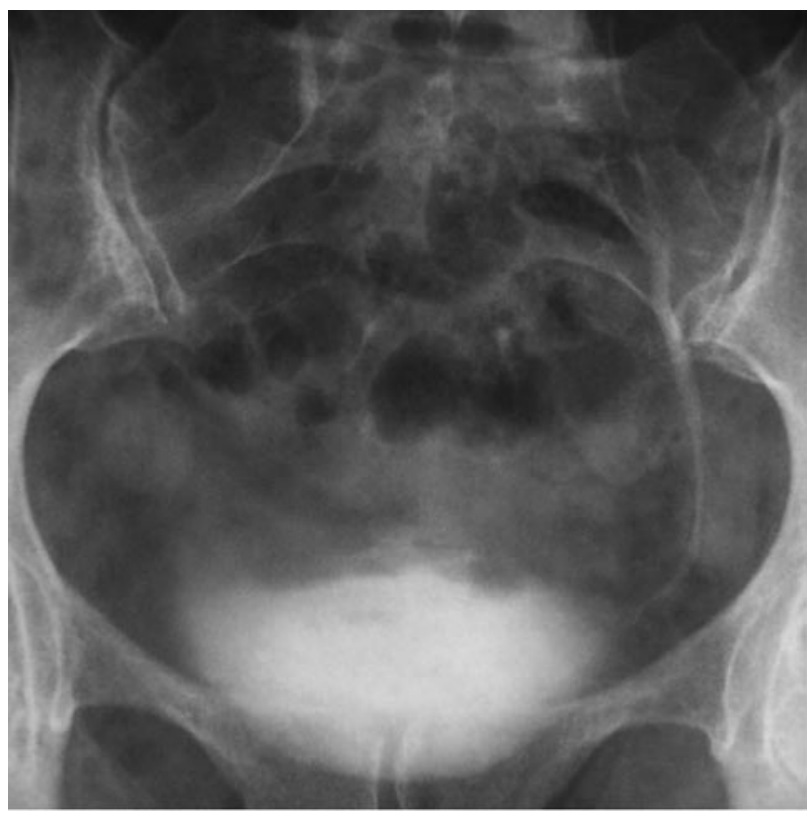

FIGURA 9. UIV de control despues de la realización de una endoureterotomía con láser en una estenosis ureteral izquierda de origen tuberculoso.
Una mala función renal homolateral, inferior al $25 \%$, pronostica una falta de respuesta a una endoureterotomía (5).

Las complicaciones más frecuentes relacionadas con la ureteroscopia y el empleo del láser de Holmium:YAG se clasifican en:

1) Intraoperatorias graves: avulsión, invaginación y perforación grave (requiere intervención quirúrgica).

2) Intraoperatorias leves: perforación leve, acceso falso, abrasión de la mucosa, extravasación, lesión térmica, hemorragia, mal funcionamiento del equipo y acceso difícil.

3) Postoperatorias graves: infección/sepsis, calle litiásica, estenosis. 4) Postoperatorias leves: obstrucción ureteral limitada y reflujo vesicoureteral. La tasa de complicaciones intraoperatorias es muy baja, estimada entre el 1-5\% (12). En un estudio multicéntrico, que compila más de 8.000 procedimientos ureteroscópicos, se describen las siguientes incidencias de complicaciones: perforación grave $(0.4 \%)$, perforación leve (1.6\%), abrasión de la mucosa $(3.7 \%)$, hemorragia $(0.8 \%)$ y estenosis $(0.5 \%)(16)$.

De acuerdo con los estudios de Davis y cols. $(7,8)$, se recomienda la colocación de un stent ureteral tras la intervención. Este debe tener un calibre mínimo de $6 \mathrm{Fr}$, y mantenerse durante al menos 6 semanas, que es el tiempo estimado que tarda en regenerarse la capa muscular ureteral. Otros autores recomiendan, sin embargo, catéteres de mayor calibre, hasta $12 \mathrm{Fr}$, durante el mismo periodo (17).

A la hora de realizar la incisión sobre la pared ureteral, ésta debe ser completa, actuándose sobre todas las capas del uréter hasta visualizar la grasa periureteral. 
Además siempre hay que tener en cuenta las relaciones del uréter con las estructuras vecinas, especialmente las vasculares. Así, en los casos de sección de uréter lumbar, es conveniente su realización en su porción ínfero-externa para evitar lesionar los vasos gonadales. En el uréter ilíaco debemos realizarla a nivel superior, al igual que en el uréter yuxtavesical, mientras que en el uréter sacro se recomienda a nivel medial para evitar los vasos uterinos.

Algunos autores, no obstante recomiendan la realización rutinaria de pruebas de imagen específicas preoperatorias, para mostrar la localización exacta de las estructuras vasculares periféricas al uréter/pélvis renal. Se recomiendan la TAC helicoidal y la ultrasonografía endoluminal, siendo esta última, la de mayor sensibilidad para detectar vasos cruzados, con baja invasividad (18).

\section{CONCLUSIÓN}

El láser de Holmium:YAG es actualmente una herramienta fundamental para el tratamiento de múltiples patologías en urología como: litiasis, HBP y, como se ha explicado anteriormente, las estenosis del aparato urinario. Su efectividad y fácil manejo, permiten obtener una elevada tasa de éxitos, con resolución de la patología estenótica, y una muy baja tasa de complicaciones. En la actualidad debe considerarse a la endoureterotomía retrógrada con láser de Holmium:YAG en la primera línea del tratamiento de las estenosis ureterales de tipo benigno (19).

La principal limitación del láser de Holmium:YAG reside en su elevado coste. Sin embargo la versatilidad de su uso en urología, e incluso en otras especialidades, hacen que, en realidad, se presente como una herramienta con una relación coste-efectividad muy favorable. Además, existen equipos de menor potencia (10-20W), que tienen un precio en el mercado más asequible.

De forma experimental, se ha publicado el uso de nuevos tipos de láser en urología. Se trata del láser de Thulium y el Erbium:YAG, los cuales podrían presentar algunas ventajas sobre el Holmium:YAG, menor tamaño e incisión más precisa sobre los tejidos, entre otras. No obstante debe aprobarse su uso en la práctica clínica para realizar estudios que lo corroboren $(20,21)$.

\section{BIBLIOGRAFÍA y LECTURAS RECOMENDADAS (*lectura de interés $y^{* *}$ lectura fundamental)}

*1. PATEL, R.C.; NEWMAN, R.C.: "Tratamiento ureteroscópico de estenosis ureterales y ureteroentéricas". Clínicas Urológicas de Norteamérica. Ed: McGrawHill Interamericana, 1: 97, 2004.

*2. HIBI, H.; MITSUI, K.; TAKI, T. y cols.: "Holmium laser incision technique for ureteral stricture using a small-caliber ureteroscope". JSLS, 4: 215, 2000.

3. WATTERSON, J.D.; SOFER, M.; WOLLIN, T.A. y cols.: "Holmium: YAG laser endoureterotomy for ureterointestinal strictures". J. Urol., 167: 1692, 2002.
4. YAMADA, Y.; HONDA, N.; HIBI, H. y cols.: "Holmium: YAG laser endoureterotomy in the treatment of ureteroenteric stricture following Indiana urinary diversion". Int. J. Urol., 8: 326, 2001.

*5. GONZÁLEZ MARTÍN, M.; CHANTADA ABAL, V.: "Endourología del aparato urinario superior". Tratado de Urología. Ed: Prous Science, 120: 2381, 2006.

*6. LEVI, J.B.; VAN ARSDALEN, K.N.: "Ureteral an ureteroenteral strictures". AUA Update Series, vol 13, lesson 29. Houston: American Urological Association, 1994.

7. DAVIS, D.M.: "Intubated ureterotomy, a new operation for ureteral and ureteropelvis stricture". Surg. Gynecol. Obstet., 76: 513, 1943.

8. DAVIS, D.M.; STRONG, G.H.; DRAKE, W.M.: "Intubated ureterotomy: Experimental work and clinical results". J. Urol., 59: 851, 1948.

9. WOLLIN, T.A.; DENSTEDT, J.D.: "The holmium laser in urology". J. Clin. Laser Med. Surg., 16: 13, 1998.

**10. LANE, B.R.; DESAI, M.M.; HEGARTY, N.J. y cols.: "Long-term efficacy of holmium laser endoureterotomy for benign ureteral strictures". Urology, 67: 894, 2006.

**11. HIBI, H.; OHORI, T.; TAKI, T. y cols.: "Long-terms results of endoureterotomy using a holmium laser". Int. J. Urol., 14: 872, 2007.

12. SINGAL, R.K.; DENSTEDT, J.D.; RAVZI, H.A. y cols.: "Holmium: YAG laser endoureteterotomy for treatment of ureteral stricture". Urology, 50: 875, 1997.

13. MAZO, E.B.; CHEPUROV, A.K.; ZENKOV, S.S. y cols.: "Ho-YAG laser endoscopic treatment of ureteral strictures". Urologia, 2: 25, 2000.

*14. SOFER, M.; BINYAMINI, J.; EKSTEIN, P.M. y cols.: "Holmium laser ureteroscopic treatment of various pathologic features in pediatrics". Urology, 69: 566, 2007.

15. FUTAO, S.; WENTONG, Z.; YAN, Z. y cols.: "Application of endoscopic Ho: YAG laser incision technique treating urethral strictures and urethral atresias in pediatric patients". Pediatr. Surg. Int., 4 : 29, 2006.

16. JONSON, D.B.; PEARLE, M.S.: "Complicaciones de la ureteroscopia”. Clínicas Urológicas de Norteamérica. Ed: McGraw-Hill Interamericana, 1: 143, 2004.

17. WOLF, J.S. Jr.; ELASHRY, O.M.; CLAYMAN, R.V.: "Long-term results of endoureterotomy for benign ureteral and ureteroenteric strictures". J. Urol., 158: 759, 1997.

*18. HENDRIKX, A.J.; NADORP, S.; DE BEER, N.A. y cols.: "The use of endoluminal ultrasonography for preventing significant bleeding during endopyelotomy: Evaluation of helical computed tomography vs endoluminal ultrasonography for detecting crossing vessels". BJU Int., 97: 786, 2006.

19. KOBAYASHI, T.; NISHIZAWA, K.; WATANABE, J. y cols.: "Endopyeloureterotomy using the holmium: YAG laser for the management of adult ureteral and ureteropelvic junction obstructions". Urol. Int., 71: 204, 2003.

20. FRIED, N.M.; MURRAY, K.E.: "High-power thulium fiber laser ablation of urinary tissues at 1.94 microm". J. Endourol., 19: 25, 2005.

21. FRIED, N.M.; TESFAYE, Z.; ONG, A.M. y cols.: "Optimization of the Erbium: YAG laser for precise incision of ureteral and urethral tissues: In vitro and in vivo results". Laser Surg. Med., 33: 108, 2003. 